place (Westminster Abbey). Yet those who opposed Newton must also be remembered: Charles Darwin refused to be buried beside Newton in Westminster Abbey; Einstein challenged Newton's physics; and John Maynard Keynes and his economics colleagues exclaimed, as Fara writes, that "Newton was not the first great scientist, but the last great magician".

Magic and science have never been easy bedfellows, despite pronouncements about their historical proximity in the early modern world. The images of magicians and scientists are different, and image-making within national settings is what this book is all about. Yet as time unfolds it becomes increasingly less important to enquire about the degree to which a particular scientist amounted to pure, disembodied genius, let alone to explore the quantum of his magic. We can assess Einstein's achievements, for example, without resorting to such extremes. If he was a genius, he was also an impoverished German Jewish immigrant who fled persecution to America. Nor do we worry whether he was unrivalled. The high-stakes, image-making process has been transferred from scientists to athletes, movie stars and politicians.

But there is one more turn of the screw. As Fara writes, "this duality as both an insane genius and a dispassionate scientist is unique to Newton". Unique it may be, but as time passes the possibility of an integrated image of Newton grows ever more remote, and in a century's time a unified view of Newton will appear even more elusive than it is now. This may not be so bad, or surprising: the lives of even the greatest scientists are no more rational or coherent than they are for the the rest of us. They never were and never will be.

George Rousseau, former regius professor of English at King's College, Aberdeen, is research professor in the School of Humanities, De Montfort University, Leicester LE1 9BH, UK.

\section{Untangling quantum mechanics}

\section{Entanglement: The Greatest Mystery in Physics \\ by Amir Aczel \\ Four Walls Eight Windows/John Wiley: 2002. 320 pp. $\$ 25 / £ 16.99$}

\section{Vlatko Vedral}

Popularizing physics is no easy task. The basic laws of quantum mechanics in particular contain many counterintuitive features that lay readers might find difficult to understand. So, in order to convey the message, there is often a tendency to oversimplify facts to the point of making them incorrect. Regrettably, this makes quantum physics much more mysterious than it really is.
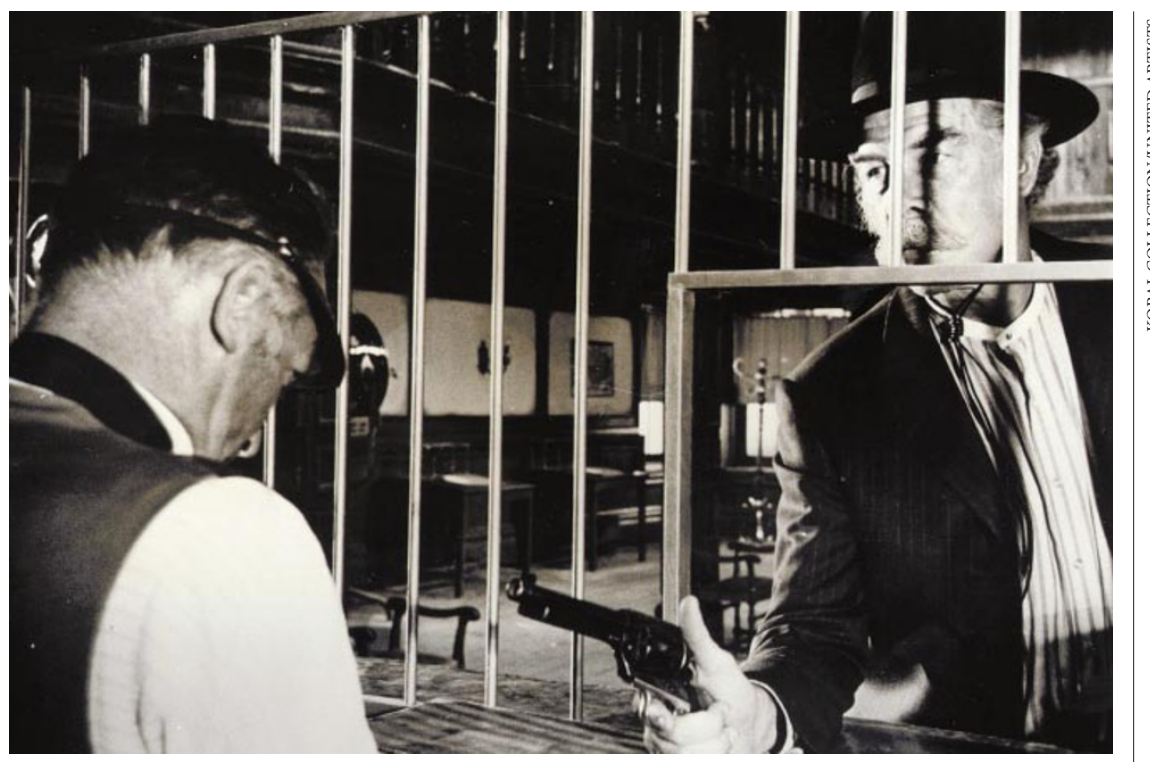

Dead or alive? The state of the cashier is correlated with whether Lee Marvin's gun has been fired.

Amir Aczel falls prey to this when describing entanglement, a key feature of quantum mechanics, in his new book. As many other authors have done, he emphasizes that when two quantum systems are entangled, and then we measure one of the systems, we immediately know (or affect) the state of the other, no matter how far apart the systems are. We are led to believe that this is somehow weird.

However, in the day-to-day world that is well described by classical newtonian physics, we often encounter correlations. For example, imagine you are observing a bank robbery. The robber is pointing a gun at the terrified bank clerk. By looking at the clerk you can tell whether the gun has gone off or not. If the clerk is alive and unharmed, you know that the gun has not fired. If the clerk is lying dead, you know that the gun has fired. There is therefore a direct correlation between the state of the gun and the state of the clerk. 'Gun fired' means 'clerk dead', and 'gun not fired' means 'clerk alive'. Of course, we assume that the robber shoots to kill and never misses.

The key (and surprising) observation is that quantum-mechanical systems may be further correlated such that the gun is in the superposition 'fired and not fired', and the clerk is then in the state 'dead and alive' at the same time! Quantum mechanically, as a consequence of this superposition, there is simply more correlation between atoms or photons than we would expect classically. This kind of quantum 'supercorrelation', first quantified precisely by John Bell in 1964 , is what we refer to as 'entanglement'.

In the early days of quantum mechanics there were several attempts to use entanglement to uncover a paradox in the foundations of quantum physics. However, by quantifying these correlations, Bell took them out of the philosophical realm of Einstein and Niels Bohr, and demonstrated how entanglement and the completeness of quantum mechanics could coexist. These extra correlations are 'real', in that they have been confirmed by experiment and, more importantly, have been successfully applied to quantum teleportation (which would otherwise be impossible) and to quantum cryptography.

The oversimplification of entanglement notwithstanding, I find that Aczel has done a good job of describing its colourful history, as well as modern developments such as teleportation. But what he perhaps does best is enlighten us with some snippets about the key physicists involved. For example, I found many interesting and humorous anecdotes about Erwin Schrödinger's love life, about John von Neumann being perceived as an alien by the US public, and about the friendship between Heisenberg and Bohr. There is, however, a dangerous tendency in popular science to idolize scientists beyond justification, making them appear more extraordinary than they really are. This book walks a fine line between historical accuracy and fictional infatuation.

I am altogether happy that there is now finally a book on entanglement, almost 70 years after its discovery, and recommend it to people interested in the historical background and practical implications of quantum mechanics. I am afraid, however, that anyone interested in understanding quantum mechanics, and the way in which scientists think about it, is much better off reading any of Richard Feynman's popular accounts, such as QED: The Strange Theory of Light and Matter (Princeton University Press, 1985). Vlatko Vedral is at the Blackett Laboratory,

Imperial College, Prince Consort Road, London SW7 2BZ, UK. 